\title{
Oral Lactobacillus Counts Predict Weight Gain Susceptibility: A 6-Year Follow-Up Study
}

\author{
Johanne Aviaja Rosing ${ }^{a} \quad$ Karen Christina Walker ${ }^{a} \quad$ Benjamin A.H. Jensen ${ }^{b}$ \\ Berit L. Heitmann a, c, d, e \\ a Department of Public Health, Section for General Practice, Faculty of Health and Medical \\ Sciences, University of Copenhagen, Copenhagen, Denmark; ${ }^{b}$ Department of Biology, \\ Laboratory for Genomics and Molecular Biomedicine, Faculty of Science, University of

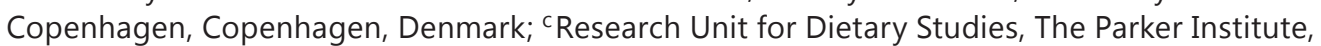 \\ Bispebjerg and Frederiksberg Hospital, The Capital Region and University of Copenhagen, \\ Frederiksberg, Denmark; ${ }^{\mathrm{d}}$ The Boden Institute of Obesity, Nutrition, Exercise \& Eating \\ Disorders Sydney Medical School, The University of Sydney, Sydney, Australia; ${ }^{\mathrm{e} N a t i o n a l}$ \\ Institute of Public Health, University of Southern Denmark, Copenhagen, Denmark
}

\section{Keywords}

Obesity $\cdot$ Human gut $\cdot$ Lactobacillus $\cdot$ Weight change $\cdot$ Prospective observational study

\begin{abstract}
Background: Recent studies have shown an association between weight change and the makeup of the intestinal microbiota in humans. Specifically, Lactobacillus, a part of the entire gastrointestinal tract's microbiota, has been shown to contribute to weight regulation. Aim: We examined the association between the level of oral Lactobacillus and the subsequent 6-year weight change in a healthy population of 322 Danish adults aged 35-65 years at baseline. Design: Prospective observational study. Results: In unadjusted analysis the level of oral Lactobacillus was inversely associated with subsequent 6-year change in BMI. A statistically significant interaction between the baseline level of oral Lactobacillus and the consumption of complex carbohydrates was found, e.g. high oral Lactobacillus count predicted weight loss for those with a low intake of complex carbohydrates, while a medium intake of complex carbohydrates predicted diminished weight gain. A closer examination of these relations showed that BMI change and Lactobacillus level was unrelated for those with high complex carbohydrate consumption. Conclusion: A high level of oral Lactobacillus seems related to weight loss among those with medium and low intakes of complex carbohydrates. Absence, or a low level of oral Lactobacillus, may potentially be a novel marker to identify those at increased risk of weight gain.

(c) 2017 The Author(s)

Published by S. Karger GmbH, Freiburg
\end{abstract}

Johanne Aviaja Rosing and Karen Christina Walker shared first authorship. 
Rosing et al.: Oral Lactobacillus Counts Predict Weight Gain Susceptibility: A 6-Year Follow-Up Study

\section{Introduction}

Too much food and too little exercise are generally considered the major reasons for obesity. However, the factors leading to obesity are clearly more complex, and several putative causes have lately been proposed, among them that the microbiota of the human gut play a role in human metabolism and the regulation of weight [1]. Studies have shown that a microbiota stemming from a lean individual has an increased capacity to break down and ferment polysaccharides compared to the capacity of a microbiota stemming from an obese individual, and that this quality of the 'lean microbiota' is associated with lower body weight and lower incidence of adiposity [2]. Lactobacillus inhabits the entire gastrointestinal tract. Previous studies have shown that certain Lactobacillus species are associated with weight gain while others are associated with protection against obesity [3].

A number of possible biological mechanisms have been proposed as a theoretical background for such associations. All fats and most protein are digested and absorbed before reaching the large intestine, as are the carbohydrates sucrose, lactose, and starch [4]. However, the more complex carbohydrates are also fermented and degraded by colonic bacteria. Hence, Drissi et al. [4] compared the genomes of Lactobacillus strains associated with weight gain and weight protection, and found that the Lactobacillus species associated with weight protection to a greater extent were involved in carbohydrate transport and metabolism than those Lactobacillus species associated with weight gain.

Sampling directly from the small intestine is challenging, and collection of fecal samples is obtained with some difficulty and low compliance of patients, thus warranting novel sampling strategies. Therefore, it is of great interest to examine if oral microbiota, easily collected from saliva with great compliance of patients, may be associated with weight change and thus may potentially be used as a marker to identify who is at risk of later weight gain and development of obesity. In support of the oral microbiota as a novel sampling site, Wang et al. [5] recently published concordance between gut and oral microbiomes in both controls and patients of rheumatoid arthritis, suggesting overlap in abundance and function of species at different body sites. Indeed, the sum of bacteria in the oral subgingival biofilm have previously been associated with obesity in adolescents [6]. Here we examine whether salivary Lactobacillus count may serve as a predictor for subsequent human weight change over a 6-year period.

\section{Subjects and Methods}

\section{Subjects}

The study was part of the Danish MONICA project and was carried out in collaboration with the Glostrup Population Studies in Copenhagen. Originally, 4,581 Danish citizens aged 35, 45, 55, and 65 years and living in 11 municipalities in the Western part of Copenhagen County, were selected, as a random sex- and agestratified sample, from the Central Person Register, and asked to participate in a general health examination in 1982/1983. Participants not born in Denmark were excluded. In total 3,608 participated. This group has been described in detail elsewhere [7]. All individuals who were still alive $(\mathrm{N}=3,498)$ were re-invited to participate in another health examination in 1987/1988 (GENMONICA) ( $=2,987$ participated) and again in 1993/1994 (MONI10) ( $\mathrm{N}=2,556$ participated). The subpopulation in the present study included a random subset (every 6th) of the 3,498 originally selected ( $\mathrm{N}=552$ ). This group was invited to a diet history interview in 1987/1988 (the present baseline). In addition, 69 subjects, who had developed gallstones between $1982 / 1983$ and 1987/1988, identified by an ultrasound examination in 1987/1988 [7], were invited to the diet history interview. Most were unaware that they had gallstones as most stones were without symptoms [7]. To examine if there was an association between dietary carbohydrates and oral bacteria, subjects agreed to give saliva and have their oral Lactobacillus cultivated.

The three health examinations included measurements of height and weight and questionnaires on lifestyle including socioeconomic positioning, smoking, and alcohol consumption. 
Rosing et al.: Oral Lactobacillus Counts Predict Weight Gain Susceptibility: A 6-Year Follow-Up Study

The project was approved by the Ethics Committee for Copenhagen County and is in accordance with the Helsinki Declaration of 1975 as revised in 1983.

\section{Anthropometric Measurements}

All anthropometric measurements were made in accordance with the WHO standards. Height was measured to the nearest $0.5 \mathrm{~cm}$ with subjects standing without shoes, heels together and head in horizontal Frankfurter plane. Body weight was measured to the nearest $0.1 \mathrm{~kg}$ using a SECA scale, with subjects wearing only underwear. BMI was calculated from mass $(\mathrm{kg}) /$ height $(\mathrm{m})^{2}$. Six-year weight changes were calculated based on the difference between measurements made at follow-up and at baseline.

\section{Questionnaire}

Subjects were asked to complete multiple questionnaires at baseline before the general health examination. From the questionnaires information on socioeconomic positioning, smoking, and alcohol consumption was retrieved. Socioeconomic position was measured by number of attended school years. Alcohol consumption was calculated as the mean consumption of units on a weekly basis. Smoking was included as a binary variable reflecting the current smoking status, yes or no. Furthermore subjects stated whether they had taken antibiotics in the past 5 years.

The same trained dentist assessed dental hygiene (poor, medium, or good) and whether there was active caries present (yes/no).

Diet

The same trained dietician interviewed all subjects about their eating habits (i.e. meal patterns, dishes, and foods) by the use of a diet history interview, based on information from the previous month. The quantity of foods and meals consumed were assessed using food models, photo series, cups and measurements. This has been described in detail previously [8]. The DANKOST program, which is derived from the Danish food composition tables, was used to calculate daily nutrient intakes and total energy intake [9]. The intake of complex carbohydrates was calculated from the total carbohydrate intake by subtracting the intake of sugars. Intake of both complex carbohydrates and sugars were measured as g/day. Energy intake was measured as $\mathrm{MJ} /$ day.

\section{Lactobacillus}

Paraffin-stimulated whole saliva was collected among individuals who were all appearing for examination after an overnight fast. Saliva was plated on Rogosa agar selective for Lactobacillus agar, which is the standard method of determining the oral Lactobacillus level [10]. The agar plates were incubated aerobically at $37^{\circ} \mathrm{C}$ for 3 days. The formation of other oral bacteria colonies is suppressed due to the acidic environment of the medium ( $\mathrm{ph}=5.4$ ), and hence all colonies formed were considered to be Lactobacillus. The number of Lactobacillus colonies can vary from 0 to approximately. 1 million colonies $/ \mathrm{ml}$. saliva. The number of Lactobacillus colonies was divided into 7 groups as follows; $10^{0}, 10^{1}, 10^{2}, 10^{3}, 10^{4}, 10^{5}, 10^{6}$. The saliva samples were obtained between 8 a.m. and 2 p.m. for all participants.

\section{Covariates}

We included the following explanatory variables in the analyses: Sex, age, socioeconomic position, BMI at baseline, alcohol intake, smoking, complex carbohydrate intake, sugar intake, energy intake, antibiotics, dental carries, and dental hygiene. Age was divided in four groups based on the four different birth years reflecting the cohorts.

\section{Statistical Methods}

We used general linear models to examine the association between oral Lactobacillus colonies and 6-year weight change. Initially, a model of oral Lactobacillus, baseline BMI, and change in BMI was tested. Subsequently, we adjusted for sex, age, and number of years in school, and baseline alcohol intake, smoking, complex carbohydrate intake, sugar intake, BMI, energy intake, and a variable indicating whether a participant had newly developed gallstones. Model fit was tested by including all continuous variables as normal, squared, and cubed variables. After a stepwise removal of insignificant covariates, all two-way interactions between oral Lactobacillus counts and the remaining covariates sex, age, total energy intake, and complex carbohydrate intake were included in the analysis by adding product terms to the model. Furthermore, because of the correlation between energy and carbohydrates all general linear models were performed 
Rosing et al.: Oral Lactobacillus Counts Predict Weight Gain Susceptibility: A 6-Year Follow-Up Study

Table 1. Characteristics of the 322 participating subjects by oral Lactobacillus count

\begin{tabular}{|c|c|c|c|c|c|c|}
\hline & \multicolumn{4}{|c|}{ Oral Lactobacillus count } & \multirow[t]{2}{*}{ P value } & \multirow[t]{2}{*}{ Total } \\
\hline & $\leq 10^{3}$ & $10^{4}$ & $10^{5}$ & $10^{6}$ & & \\
\hline Male, N (\%) & $59(47.2)$ & $32(47.8)$ & $35(55.6)$ & $36(53.7)$ & & $162(50.3)$ \\
\hline Female, N (\%) & $66(52.8)$ & $35(52.2)$ & $28(44.4)$ & $31(46.3)$ & & $160(49.7)$ \\
\hline \multicolumn{7}{|l|}{ Age (years), N (\%) } \\
\hline 35 & $14(11.2)$ & $20(29.9)$ & $16(25.4)$ & $21(31.3)$ & & $71(22.0)$ \\
\hline 45 & $29(23.2)$ & $19(28.4)$ & $12(19.0)$ & $22(32.8)$ & & $82(25.5)$ \\
\hline 55 & $36(28.8)$ & $15(22.4)$ & $22(34.9)$ & $18(26.9)$ & & $91(28.3)$ \\
\hline 65 & $46(36.8)$ & $13(19.4)$ & $13(20.6)$ & $6(9.0)$ & & $78(24.2)$ \\
\hline \multicolumn{7}{|l|}{ Education (years), N (\%) } \\
\hline$\leq 7$ & $25(20.0)$ & $18(26.9)$ & $18(28.6)$ & $22(32.8)$ & & $83(25.8)$ \\
\hline 8-11 & $59(47.2)$ & $28(41.8)$ & $31(49.2)$ & $35(52.2)$ & & $153(47.5)$ \\
\hline$>11$ & $41(32.8)$ & $21(31.3)$ & $14(22.2)$ & $10(14.9)$ & & $86(26.7)$ \\
\hline \multicolumn{7}{|l|}{ Smoking, N (\%) } \\
\hline Non-smoker, N (\%) & $93(74.4)$ & $35(52.2)$ & $33(52.4)$ & $25(37.3)$ & & $186(57.8)$ \\
\hline Smoker, N (\%) & $32(25.6)$ & $32(47.8)$ & $30(47.6)$ & $42(62.7)$ & & $136(42.2)$ \\
\hline BMI baseline, $\mathrm{kg} / \mathrm{m}^{2}$ (mean $\pm \mathrm{SD}$ ) & $24.8 \pm 4.1$ & $24.8 \pm 3.8$ & $25.6 \pm 3.7$ & $25.2 \pm 3.1$ & 0.54 & $25.1 \pm 3.8$ \\
\hline Alcohol, units/week (mean \pm SD) & $8.6 \pm 13.2$ & $7.6 \pm 11.1$ & $8.8 \pm 11.4$ & $10.1 \pm 13.3$ & 0.70 & $8.8 \pm 12.5$ \\
\hline Carbohydrates, g/day (mean \pm SD) & $207.2 \pm 79.1$ & $191.5 \pm 64.6$ & $223.8 \pm 84.5$ & $214.0 \pm 73.4$ & 0.10 & $208.6 \pm 76.6$ \\
\hline Complex carbohydrates, g/day (mean \pm SD) & $179.6 \pm 64.1$ & $164.4 \pm 54.2$ & $188.3 \pm 65.9$ & $179.9 \pm 56.7$ & 0.16 & $178.3 \pm 61.0$ \\
\hline Sugar intake, g/day $($ mean \pm SD) & $27.7 \pm 26.2$ & $27.1 \pm 28.3$ & $35.5 \pm 39.7$ & $34.1 \pm 30.2$ & 0.21 & $30.4 \pm 30.6$ \\
\hline Protein intake, g/day (mean \pm SD) & $73.1 \pm 22.1$ & $69.9 \pm 21.5$ & $76.6 \pm 25.8$ & $73.4 \pm 20.3$ & 0.41 & $73.2 \pm 22.4$ \\
\hline Fat intake, g/day (mean $\pm \mathrm{SD})$ & $90.9 \pm 32.0$ & $85.2 \pm 33.1$ & $97.7 \pm 39.2$ & $97.4 \pm 33.0$ & 0.10 & $92.4 \pm 34.1$ \\
\hline Total energy intake, $\mathrm{MJ} /$ day $($ mean $\pm \mathrm{SD}$ ) & $8.7 \pm 3.0$ & $8.1 \pm 2.6$ & $9.3 \pm 3.2$ & $9.1 \pm 2.6$ & 0.07 & $8.7 \pm 2.9$ \\
\hline Change in BMI, $\mathrm{kg} / \mathrm{m}^{2}($ mean $\pm \mathrm{SD})$ & $1.0 \pm 1.8$ & $1.1 \pm 1.4$ & $0.9 \pm 1.7$ & $0.3 \pm 1.9$ & 0.03 & $0.9 \pm 1.8$ \\
\hline
\end{tabular}

using residual energy adjustment, by regressing complex carbohydrate intakes of the individuals on their total energy intakes.

Sensitivity analyses were completed by including each covariate in the final model, when possible both as continuous and as categorical variables. ANOVA was used to examine potential differences related to participation. Regression coefficients were considered statistically significant for values of $p<0.05$. All statistical analyses were carried out with IBM SPSS Statistics version 23 (Armonk, NY, USA).

\section{Results}

A total of 435 accepted the invitation to participate and completed a diet history interview (see flowchart). Complete data on anthropometric measures, oral Lactobacillus levels, and questionnaire information were collected at baseline from 390 of these subjects. One male subject reported consuming 914 drinks of alcohol per week, and was excluded from further analysis. At follow-up anthropometric measurements were collected from a total of 322 subjects, who were considered to be the participants. There were no differences in sex, age, or BMI measured in 1982/1983 between the 322 participants and the larger cohort of 3,608 subjects. There were also no differences between participants $(\mathrm{n}=322)$ and non-participants $(\mathrm{n}=113)$ in sex, age, or BMI in $1987 / 1988$. Also there were no differences in oral Lactobacillus count, complex carbohydrate intake, or total energy intake between the 68 subjects who completed all examinations in 1987/1988 but did not complete the anthropometric measurements in 1993/1994, and the 322 subjects who completed all examinations in both 1987/1988 and 1993/1994. Finally, there were no differences in sex, age, baseline BMI, Lactobacillus count, intake of complex carbohydrates or energy, 6-year change in BMI, 
Table 2. Crude and adjusted associations between total energy or nutrients and subsequent 6-year change in BMI $\left(\mathrm{kg} / \mathrm{m}^{2}\right)$
Rosing et al.: Oral Lactobacillus Counts Predict Weight Gain Susceptibility: A 6-Year Follow-Up Study

\begin{tabular}{lll}
\hline & \multicolumn{2}{l}{ Change in BMI, $\beta \pm$ SE } \\
\cline { 3 - 3 } & crude & adjusted* \\
\hline Energy, MJ/day & $-0.000028 \pm 0.000034$ & $-0.000038 \pm 0.000041$ \\
CHO, g/day & $-0.001 \pm 0.002$ & $-0.001 \pm 00.2$ \\
Sugar, g/day & $-0.001 \pm 0.003$ & $-0.003 \pm 0.003$ \\
Alcohol, U/day & $0.04 \pm 0.008$ & $0.007 \pm 0.008$ \\
Fat, g/day & $-0.004 \pm 0.003$ & $0.000 \pm 0.00009$ \\
Protein, g/day & $-0.002 \pm 0.004$ & $0.000 \pm 0.000$ \\
\hline
\end{tabular}

*Adjusted for sex, age, socioeconomic position, BMI at baseline and smoking. Statistical test: General linear model.

Table 3. Characteristics of the subjects by intake of complex carbohydrates (unadjusted values)

\begin{tabular}{|c|c|c|c|c|c|c|}
\hline & \multicolumn{6}{|c|}{ Complex carbohydrate intake } \\
\hline & \multicolumn{2}{|c|}{$\begin{array}{l}\text { lower third } \\
\text { (56-145 g/day) }\end{array}$} & \multicolumn{2}{|c|}{$\begin{array}{l}\text { middle third } \\
(146-191 \mathrm{~g} / \text { day })\end{array}$} & \multicolumn{2}{|c|}{$\begin{array}{l}\text { upper third } \\
(192-484 \mathrm{~g} / \text { day })\end{array}$} \\
\hline & $\mathrm{n}$ & mean (SE) & $\mathrm{n}$ & mean (SE) & $\mathrm{n}$ & mean (SE) \\
\hline Lactobacillus count $10^{0}-10^{3}$ & 38 & & 43 & & 44 & \\
\hline BMI baseline, $\mathrm{kg} / \mathrm{m}^{2}$ & & $25.5(4.5)$ & & $25.1(3.7)$ & & $24.1(4.4)$ \\
\hline BMI change, $\mathrm{kg} / \mathrm{m}^{2}$ & & $1.6(2.6)$ & & $0.8(1.5)$ & & $0.9(1.3)$ \\
\hline Energy, MJ/day & & $6.0(1.4)$ & & $8.3(1.6)$ & & $11.5(2.8)$ \\
\hline Sugar, g/day & & $14.1(13.0)$ & & $30.3(26.7)$ & & $36.8(29.7)$ \\
\hline Alcohol, units/week & & $6.0(7.5)$ & & $7.0(9.1)$ & & $12.4(18.7)$ \\
\hline Lactobacillus count $10^{4}$ & 31 & & 20 & & 16 & \\
\hline BMI baseline, $\mathrm{kg} / \mathrm{m}^{2}$ & & $26.1(4.1)$ & & $23.0(2.5)$ & & $24.5(3.7)$ \\
\hline BMI change, $\mathrm{kg} / \mathrm{m}^{2}$ & & $1.3(1.6)$ & & $1.3(1.4)$ & & $0.8(0.9)$ \\
\hline Energy, MJ/day & & $6.3(1.6)$ & & $8.4(1.5)$ & & $11.3(2.1)$ \\
\hline Sugar, g/day & & $27.7(35.9)$ & & $20.2(12.2)$ & & $34.7(25.0)$ \\
\hline Alcohol, units/week & & $7.4(12.0)$ & & $6.8(11.0)$ & & $9.1(10.2)$ \\
\hline Lactobacillus count $10^{5}$ & 18 & & 21 & & 24 & \\
\hline BMI baseline, $\mathrm{kg} / \mathrm{m}^{2}$ & & $27.1(3.7)$ & & $25.2(4.1)$ & & $24.9(3.3)$ \\
\hline BMI change, $\mathrm{kg} / \mathrm{m}^{2}$ & & $0.8(1.7)$ & & $1.6(2.2)$ & & $0.5(1.0)$ \\
\hline Energy, MJ/day & & $6.9(1.1)$ & & $8.8(2.1)$ & & $11.6(3.6)$ \\
\hline Sugar, g/day & & $21.2(18.0)$ & & $45.0(57.8)$ & & $37.8(29.3)$ \\
\hline Alcohol, units/week & & $8.6(8.5)$ & & $6.7(11.1)$ & & $10.8(13.6)$ \\
\hline Lactobacillus count $10^{6}$ & 20 & & 24 & & 23 & \\
\hline BMI baseline, $\mathrm{kg} / \mathrm{m}^{2}$ & & $26.0(3.3)$ & & $25.5(3.1)$ & & $24.1(2.8)$ \\
\hline BMI change, $\mathrm{kg} / \mathrm{m}^{2}$ & & $-0.6(1.7)$ & & $0.7(2.3)$ & & $0.8(1.5)$ \\
\hline Energy, MJ/day & & $6.8(1.6)$ & & $8.7(1.8)$ & & $11.5(1.9)$ \\
\hline Sugar, g/day & & $23.1(20.6)$ & & $34.8(25.8)$ & & $42.9(38.4)$ \\
\hline Alcohol, units/week & & $11.2(12.8)$ & & $11.5(17.9)$ & & $7.8(7.0)$ \\
\hline
\end{tabular}

or loss to follow-up for those who had gallstones and the random subset selected for the diet interview.

Table 1 shows the characteristics of the study population $(n=322)$ according to the included covariates and the nutrients protein and fat. On average this population subset was slightly overweight $\left(\mathrm{BMI}=25.1 \pm 3.8 \mathrm{~kg} / \mathrm{m}^{2}\right)$, and gained $0.9 \pm 1.8 \mathrm{~kg} / \mathrm{m}^{2}$ over the 6 years. 
Fig. 1. The association between 6-year change in BMI and counts of oral Lactobacillus at three levels of complex carbohydrate intake. Adjusted for BMI at baseline. Statistical test: general linear model.
Rosing et al.: Oral Lactobacillus Counts Predict Weight Gain Susceptibility: A 6-Year Follow-Up Study

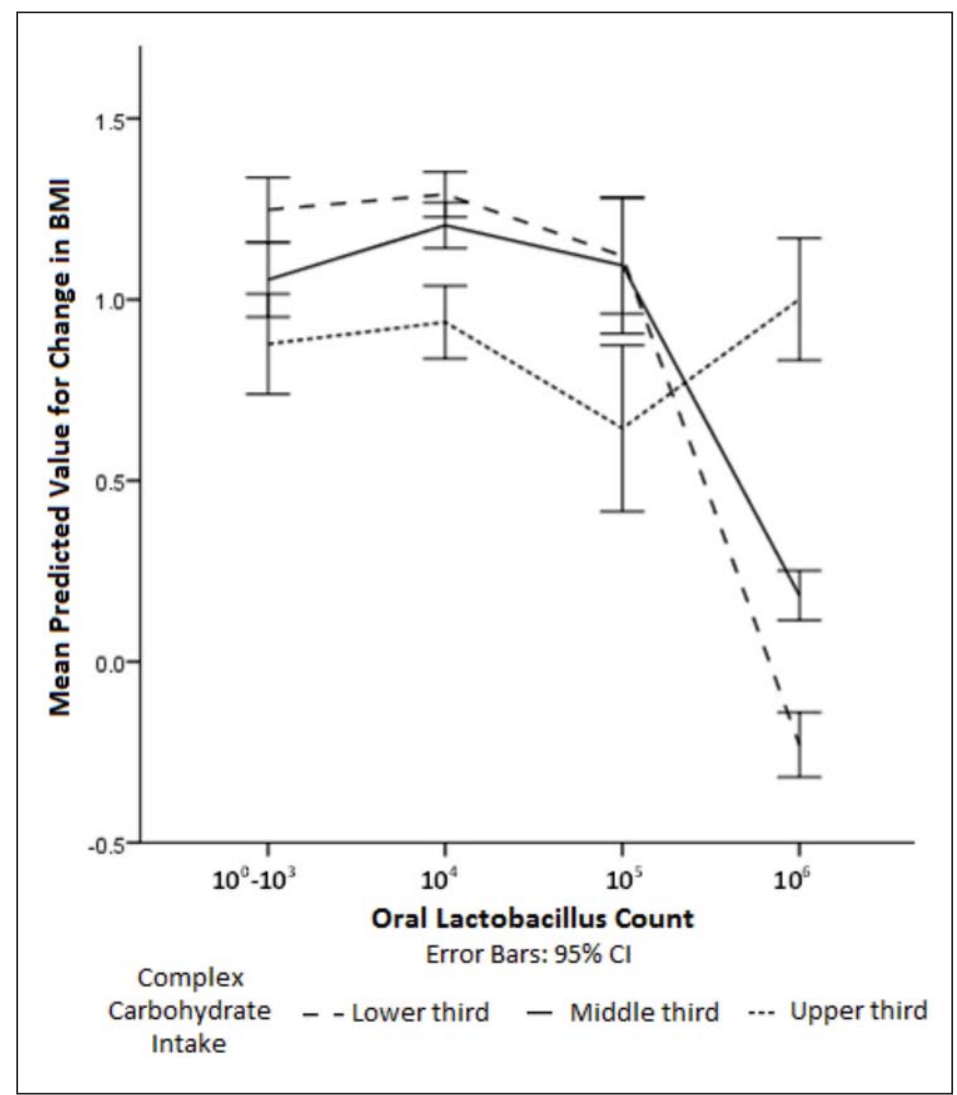

Total carbohydrate intake was $208.6 \pm 76.7 \mathrm{~g} /$ day, of which $178.3 \pm 61.0 \mathrm{~g} /$ day was from complex carbohydrates and $30.4 \pm 30.6 \mathrm{~g} /$ day from simple sugars.

The four groups with the lowest count of oral Lactobacillus were combined into one, because only few subjects were present in each group, and visual differentiation between 1 , 10,100 and 1,000 colonies was assessed with difficulty, hence, potentially leading to misplacement error. This procedure is commonly used [10], and resulted in 4 oral Lactobacillus groups: $10^{0}-10^{3}, 10^{4}, 10^{5}$, and $10^{6}$.

Table 2 shows the crude and adjusted associations between total energy or nutrients and subsequent 6-year change in BMI. All associations, both crude and adjusted, were insignificant.

Table 3 shows the characteristics of the subjects by their consumption of complex carbohydrates (g/day) and their count of oral Lactobacillus colonies. The largest proportion $(38.8 \%$, $\mathrm{n}=125)$ of the subjects had an oral Lactobacillus count of $\leq 10^{3}$ colonies while $67(20.8 \%)$ subjects had a count of $10^{6}$ oral Lactobacillus colonies.

Cross-sectional associations between BMI at baseline and counts of Lactobacillus colonies were neither significant before ( $\mathrm{p}=0.54)$ nor after adjusting for relevant covariates such as sex, age, socioeconomic position, alcohol, smoking, complex carbohydrate intake, sugar intak,e and energy intake ( $\mathrm{p}=0.28$ ) (data not shown).

An overall inverse association was found between counts of oral Lactobacillus and subsequent 6-year change in BMI $(p=0.03)$ before adjusting for covariates $\left(\leq 10^{3}: \beta=0 ; 10^{4}: \beta=0.13\right.$ $(95 \% \mathrm{CI}-0.40 ; 0.65) ; 10^{5}: \beta=-0.09(95 \% \mathrm{CI}-0.64 ; 0.44) ; 10^{6}: \beta=-0.70(95 \% \mathrm{CI}-1.23 ;-0.18)$ (data not shown). The adjusted analyses however, revealed significant interactions between counts of oral Lactobacillus colonies and complex carbohydrate intake ( $\mathrm{p}$ for interaction $=0.04$ ). 
The associations between the counts of oral Lactobacillus and the subsequent 6-year change in BMI by levels of carbohydrate intake are shown in figure 1. Analyses were adjusted for BMI at baseline.

The figure shows that the largest subsequent 6-year increase in BMI was found for those with the lowest baseline levels of Lactobacillus $\left(\leq 10^{3}\right.$ and $\left.10^{4}\right)$, and the lowest intake of complex carbohydrate, while a 6-year loss in BMI was found for those with the highest level of Lactobacillus $\left(10^{6}\right)$ and the lowest intake of complex carbohydrates. The 6-year change in BMI for those subjects consuming the highest intake of complex carbohydrates was $0.9 \pm 0.4$ $\mathrm{kg} / \mathrm{m}^{2}$ over the 6-year period, but this gain in BMI was not related to Lactobacillus level.

For those with medium intakes of complex carbohydrates, change in BMI was also similar among those with counts of oral Lactobacillus $\leq 10^{5}$ on average $1.1 \pm 0.3 \mathrm{~kg} / \mathrm{m}^{2}$ during the course of 6 years, whereas BMI remained stable $\left(0.2 \pm 0.2 \mathrm{~kg} / \mathrm{m}^{2}\right)$ for those with high counts $\left(10^{6}\right)$. The adjusted analysis also revealed a significant interaction $(\mathrm{p}=0.03)$ between counts of oral Lactobacillus colonies and total energy intake.

We performed specific sensitivity analyses to examine confounding from sugar intake and smoking, included as both a continuous and a categorical variable, but all results were essentially similar before and after inclusion of these variables (data not shown).

Also, none of the other covariates, including the indicator variable whether participants had developed gallstones, or additional variables on active caries, dental hygiene, or antibiotics use in the past 5 years, were found to influence the association between Lactobacillus and 6-year weight gain (data not shown), and none of these variables were consequently included in the final model, except for BMI at baseline.

\section{Discussion}

The present study proposes oral Lactobacillus counts as a novel biomarker for weight gain susceptibility in people with low and medium intake of complex carbohydrates. We found that high oral Lactobacillus count predicted weight loss if the intake of complex carbohydrates was low, while at medium intake of complex carbohydrates high oral Lactobacillus count $\left(10^{6}\right)$ predicted weight maintenance. There was no association between oral Lactobacillus and 6-year changes in BMI in subjects with high intakes of complex carbohydrates.

The mechanism behind our observations remains elusive, but alterations in the gut microbiota are a possible confounding cause giving the proposed interrelationship between the oral and gut microbiome [11]. Gut microbes increase energy extracting potential of otherwise indigestible nutrients [12], and have been shown to affect host metabolism and weight change in both mice [13] and humans [14]. Ridaura et al. [2] transferred fecal samples from twins discordant for obesity to germ free mice, which subsequently became either obese or remained lean, dependent on the microbiota they received. The authors additionally identified microbiota-diet interactions, which, together with our findings, suggest a complex interface between diet and gut microbiota underlying human obesity. The biology behind obesity and obesity-promoting risk factors is complex. The beneficial effect of high oral Lactobacillus counts may arise from other factors than gut microbiota. Some studies point toward anti-inflammatory properties of Lactobacillus. Indeed, in a human setting, van Baarlen et al. [15] found an anti-inflammatory potential of Lactobacillus plantarum in the small intestine. Through biopsies in the duodenal mucosa of healthy adults receiving either live or heat-killed L. plantarum, Baarlen et al. [15] demonstrated a regulation of the key inflammatory transcription factor in subjects receiving live bacteria. Supporting this immunomodulatory role of Lactobacillus, Poutahidis et al. [16] showed that Lactobacillus reuteri administered in the drinking water facilitated resistance to diet-induced obesity, despite $L$. 
reuteri failing to colonize the intestinal cavity. Subsequent experiments confirmed that $L$. reuteri induced an adipose-specific increase of anti-inflammatory regulatory $T$ cells. Thus, in our human cohort study, it is not possible to determine whether the preventative effect of increasing amounts of oral Lactobacillus on weight change is due to a differential microbial community, differences in the microbial gene expression, immune regulation, or a combined effect of several factors. We were unable to discriminate between distinct members of the Lactobacillus family. This is an obvious limitation as Lactobacillus subspecies may have different weight change potential. Numerous studies have shown a role of Lactobacillus in weight regulation where species such as Lactobacillus fermentum, Lactobacillus gasseri, Lactobacillus johnsonii and Lactobacillus crispatus associate directly with BMI [17] and weight gain [3], whereas Lactobacillus casei and L. plantarum associate with absence of obesity. In support of our findings, most species of Lactobacillus in the oral cavity belong to the L. casei group [18].

The inverse association between oral Lactobacillus and weight change remained similar before and after adjusting for numerous covariates, including sugar intake, alcohol intake and smoking, which in other studies have been found to influence both oral and intestinal Lactobacillus count as well as risk of obesity $[19,20], 21]$. A final set of sensitivity analyses included adjustment for use of antibiotics in previous 5 years, dental hygiene, and active caries. Oral Lactobacillus have previously been found to correlate with dental caries [19], and the use of antibiotics may affect the composition of the gut microbiota [22]. Data on antibiotics use in previous 5 years was available on a subset of 282 individuals. Yet results remained similar before and after such adjustment.

To our knowledge, this is the first report examining associations between oral Lactobacillus levels and subsequent 6-year weight change. Our sample included $>300$ subjects selected from a random subset of the Danish population. Participation was generally high both at baseline and at follow-up, and participants were not different from non-participants or from those lost to follow-up. Inclusion of subjects who developed gallstones did not influence the results, potentially because most of these gallstones were asymptomatic and hence unknown to the participants.

Previous studies found that some species of Lactobacillus from gut microbiota were related to weight [4]. However, most previous studies were cross-sectional and conducted on human subsamples, which were much smaller and/or restricted to clinical subsets often with obesity. Our findings suggest that some of the discrepancy in results from previous reports may depend on differences in carbohydrate intake. Armougom et al. [23] 2009 found higher levels of Lactobacillus in feces from obese individuals compared to feces from both anorexic subjects and normal-weight controls. In contrast, Karlsson et al. [24] did not find any significant association between the presence of Lactobacillus and the current BMI among 20 overweight and obese children and 20 children within the normal range of BMI. Our prospective analysis is a step towards a better understanding of the relationship between oral-cavity microbiota and subsequent host weight change as a time relationship cannot be assessed from cross-sectional studies. Professionals gathered the dietary information and performed the anthropometric measurements. Further, a dentist determined the level of oral Lactobacillus. The finding that oral Lactobacillus counts were unrelated to weight development in subjects with high intake of complex carbohydrates supports the previously reported observation of $L$. casei dominating the oral cavity [18]. L. casei is beneficial for immune regulation and weight maintenance, but fuels on simple carbohydrates [24] and may therefore not impact weight development in subjects with high intake of complex carbohydrates.

Some study limitations also need to be addressed. The dietary assessment period for this study was 1 month, which may not reflect long-term exposure. Subjects in the present study 
underreported fat- and/or carbohydrate-rich foods [8]. Especially the overweight and obese subjects gave biased information on both energy and fat and/or carbohydrate intakes. This may have created some misclassification and hence attenuated the observed differences between the three intake groups. However, despite this we were still able to identify significant associations between Lactobacillus level and weight change, thus validating the associations. The level of oral Lactobacillus is relatively stable during the course of the day, but significantly higher levels can be found in the morning before breakfast and tooth brushing, especially in subjects with high Lactobacillus [10]. All saliva samples were collected in fasted state between 8 a.m. and 2 p.m., and hence some variation can have been introduced. Again, strengthening our findings, this bias is expected to have attenuated rather than inflated our results.

We took one measurement only of the microbiota genus and no species level data, which will have introduced some measurement error. However, also this potential measurement error would tend to attenuate associations, and we found significant associations despite this. It should be noted that major advances in microbiota analyses have occurred since we cultured total Lactobacillus that would have made it possible to study other genera in the oral microbiota which limits the biological interpretability of the study. Furthermore, the observational design of the study gives rise to concerns of unmeasured confounders including physical activity habits, genetics, and more detailed dietary factors. Future studies should include information on these variables.

It can be argued that the actual difference in 6-year BMI change, which is equivalent to approximately $2.5 \mathrm{~kg}$, appear small and potentially irrelevant from a public health perspective. However, we found previously [25] that the weight gains leading to obesity are small and continuous [25] and the changes observed in the present study, which were related to low oral Lactobacillus, are in accordance with this. Thus, our results may be of importance in a public health setting while oral Lactobacillus may be of less clinical relevance.

Our sample included a random subset of participants as well as subjects with newly developed gallstones, which may limit generalizability of our results. However, as results were essentially similar with or without the gallstone patients included, the inclusion of this group may not be expected to impact on generalizability of our results.

In conclusion, the present study suggests that a high oral Lactobacillus count, found among about one-fifth of the population, might mark those who are more resistant to future weight gain, in particularly among those with a low intake of complex carbohydrates. Conversely, absence or a low level of oral Lactobacillus, may potentially be considered a marker to identify those at increased risk of weight gain. However, this conclusion should be treated with caution and validated in future studies including strain-level characterization of Lactobacillus.

\section{Authors' Contributions}

BLH designed and conducted research; JR and CW analyzed data; JR and CW had main responsibility for writing the article. BAHJ provided critical inputs to the results and discussion. BLH had primary responsibility for final content. All authors read and approved the final manuscript.

\section{Funding}

This study was supported by a grant to Berit L. Heitmann from the Danish MRC under the FREJAprogram (Female Researchers in Joint Action) 


\section{Disclosure Statement}

The authors have no conflicts of interest to declare.

\section{References}

1 Ley RE: Obesity and the human microbiome. Curr Opin Gastroenterol 2010;26:5-11.

2 Ridaura VK, Faith JJ, Rey FE, Cheng J, Duncan AE, Kau AL, et al: Cultured gut microbiota from twins discordant for obesity modulate adiposity and metabolic phenotypes in mice. Science 2013;341:1241214.

3 Million M, Angelakis E, Paul M, Armougom F, Leibovici L, Raoult D: Comparative meta-analysis of the effect of Lactobacillus species on weight gain in humans and animals. Microb Pathog 2012;53100-108.

4 Drissi F, Merhej V, Angelakis E, El Kaoutari A, Carrière F, Henrissat B, et al: Comparative genomics analysis of Lactobacillus species associated with weight gain or weight protection. Nutr Diabetes 2014;4:e109.

5 Zhang X, Zhang D, Jia H, Feng Q, Wang D, Liang D, et al: The oral and gut microbiomes are perturbed in rheumatoid arthritis and partly normalized after treatment. Nat Med 2015;21:1-13.

6 Zeigler CC, Persson GR, Wondimu B, Marcus C, Sobko T, Modéer T: Microbiota in the oral subgingival biofilm is associated with obesity in adolescence. Obesity (Silver Spring) 2012;20:157-164.

7 Heitmann BL: Body fat in the adult Danish population aged 35-65 years: an epidemiological study. Int J Obes 1991;15:535-545.

8 Heitmann BL: The influence of fatness, weight change, slimming history and other lifestyle variables on diet reporting in Danish men and women aged 35-65 years. Int J Obes Relat Metab Disord 1993;17:329-336.

9 Heitmann BL, Lissner L: Dietary underreporting by obese individuals - is it specific or non-specific? BMJ 1995; 311:986-989.

10 Thylstrup A, Fejerskov 0: (eds): Textbook of Cariology. Copenhagen, Munksgaard, 1986.

11 Arimatsu K, Yamada H, Miyazawa H, Minagawa T, Nakajima M, Ryder MI, et al: Oral pathobiont induces systemic inflammation and metabolic changes associated with alteration of gut microbiota. Sci Rep 2014;4: 4828.

12 Bäckhed F, Manchester JK, Semenkovich CF, Gordon JI: Mechanisms underlying the resistance to diet-induced obesity in germ-free mice. Proc Natl Acad Sci U S A 2007;104:979-984.

13 Turnbaugh PJ, Ley RE, Mahowald MA, Magrini V, Mardis ER, Gordon JI: An obesity-associated gut microbiome with increased capacity for energy harvest. Nature 2006;444:1027-1031.

14 Turnbaugh PJ, Hamady M, Yatsunenko T, Cantarel BL, Duncan A, Ley RE, et al: A core gut microbiome in obese and lean twins. Nature 2009;457:480-484.

15 van Baarlen P, Troost FJ, van Hemert S, van der Meer C, de Vos WM, de Groot PJ, et al: Differential NF-kappaB pathways induction by Lactobacillus plantarum in the duodenum of healthy humans correlating with immune tolerance. Proc Natl Acad Sci U S A 2009;106:2371-2376.

16 Poutahidis T, Kleinewietfeld M, Smillie C, Levkovich T, Perrotta A, Bhela S, et al: Microbial reprogramming inhibits Western diet-associated obesity. PLoS One 2013;8:e68596.

17 Karlsson FH, Tremaroli V, Nookaew I, Bergström G, Behre CJ, Fagerberg B, et al: Gut metagenome in European women with normal, impaired and diabetic glucose control. Nature 2013;498:99-103.

18 Badet C, Thebaud NB: Ecology of lactobacilli in the oral cavity: a review of literature. Open Microbiol J 2008; 2:38-48.

19 Beighton D, Adamson A, Rugg-Gunn A: Associations between dietary intake, dental caries experience and salivary bacterial levels in 12-year-old English schoolchildren. Arch Oral Biol 1996;41:271-280.

20 Sakki T, Knuuttila M: Controlled study of the association of smoking with lactobacilli, mutans streptococci and yeasts in saliva. Eur J Oral Sci 1996;104:619-622.

21 Biedermann L, Zeitz J, Mwinyi J, Sutter-Minder E, Rehman A, Ott SJ, et al: Smoking cessation induces profound changes in the composition of the intestinal microbiota in humans. PLoS One 2013;8:e59260.

22 Million M, Raoult D: The role of the manipulation of the gut microbiota in obesity. Curr Infect Dis Rep 2013 15:25-30

23 Armougom F, Henry M, Vialettes B, Raccah D, Raoult D: Monitoring bacterial community of human gut microbiota reveals an increase in Lactobacillus in obese patients and methanogens in anorexic patients. PLoS One 2009;4:e7125.

24 Cai H, Rodríguez BT, Zhang W, Broadbent JR, Steele JL: Genotypic and phenotypic characterization of Lactobacillus casei strains isolated from different ecological niches suggests frequent recombination and niche specificity. Microbiology 2007; 153:2655-2665.

25 Heitmann BL, Garby L: Patterns of long-term weight changes in overweight developing Danish men and women aged between 30 and 60 years. Int J Obes Relat Metab Disord 1999;23:1074-1078. 